\title{
6 AUSBLICK
}

Die sektorübergreifenden Prinzipien, Kriterien und Indikatoren (PKI) für die Nachhaltigkeitsbewertung der Interaktionsfelder von jagdlichen Aktivitäten, Landwirtschaft, Forstwirtschaft, und Freizeit- und Erholungsnutzungen zu Wildtieren, Wildlebensräumen und Jagd sollen im Internet zur praktischen Anwendung zur Verfügung gestellt werden. Hierzu besteht seitens des Biosphärenpark Wienerwald Managements die Bereitschaft, die PKI-Sets auf der Homepage des Biosphärenparks bereitzustellen. Grundsätzlich besteht bei entsprechender Finanzierungsbereitschaft interessierter Fördergeber auch die Möglichkeit, nach dem Vorbild der bestehenden Internet-Plattform „Nachhaltige Jagd“ (www.biodiv.at/chm/jagd/ bzw. www.biologischevielfalt.at/nachhaltige-nutzung/nachhaltige-jagd/) eine internetbasierte Möglichkeit zur interaktiven elektronischen Selbstbewertung zu schaffen. Kommentare und Verbesserungsvorschläge werden weiterhin gesammelt, um im Sinne der dynamischen Konzeption des Sets zukünftige Nachjustierungen zu ermöglichen. Größtmögliche Praktikabilität bei der Anwendung des Sets und bestmögliche Aussagekraft sollen dabei stets im Vordergrund stehen.

Der im vorliegenden Projekt gewählte methodische Ansatz der Nachhaltigkeitsbeurteilung verfolgt vor allem das Ziel, dass sich die lokal betroffenen Personen von den Prinzipien-, Kriterien- und Indikatorensets direkt angesprochen fühlen und sich durch eigenständige Verwendung dieses Beurteilungsinstrumentes mit Nachhaltigkeitsanforderungen beschäftigen. Dies ersetzt nicht die Entwicklung zusätzlicher Monitoringsysteme, die für die großräumige objektive Beurteilung der Nachhaltigkeit „von außen“ erforderlich sein können. Anzustreben ist eine Kombination des hier entwickelten, auf den „Anwender“ (Jäger, Förster, Landwirt, Freizeit- und Erholungsmanagement, Grundeigentümer) bezogenen Beurteilungsansatzes mit statistisch auswertbaren Monitoringmethoden für die Entwicklung von Wildtierarten und deren Lebensräumen (z. B. durch ein Netz repräsentativer Untersuchungsgebiete). Dadurch wäre es auch möglich, dass die Bestände oder Bestandestrends der jagdbaren und der gefährdeten Wildarten im betrachteten Gebiet erfasst und mit den überregionalen Entwicklungen verglichen werden können, um die gewonnenen Erkenntnisse bei der weiteren Managementplanung zu berücksichtigen. Überregionale Kontrollen sollten idealerweise in einem international abgestimmten Programm je nach Wildart auf übergeordneter Ebene (z. B. Populationen, bei Zugvögeln auf Flyway-Ebene) durchgeführt werden.

Die Projektergebnisse sollen zur allgemeinen Bewusstseinsbildung für integrative nachhaltige Nutzung natürlicher Ressourcen und für ökologische, ökonomische und sozio-kulturelle Vernetzungen Verwendung finden. Dazu sind eine entsprechende Öffentlichkeitsarbeit und der systematische Transport von Ergebnissen zur Basis der Nutzergruppen notwendig, ebenso wie die Integration von Projektergebnissen in bestehende Regelwerke des Biosphärenparks Wienerwald. Durch die enge Einbeziehung von Stakeholdern in der Region (Betriebe, Grundeigentümer, Landnutzervertreter, Biosphärenparkmanagement etc.), v. a. im Rahmen der projektbezogenen Partizipationsplattform, konnten hierfür günstige Voraussetzungen geschaffen werden.

Eine Weiterführung der im Projekt ISWI-MAB aufgebauten Stakeholder-Plattform als "intersektorales Forum für Konfliktmanagement" scheint möglich und wird empfohlen.

Ein MAB-Folgeprojekt (IESP) soll zur Weiterentwicklung dieses Nachhaltigkeitsansatzes und zur praktischen Umsetzung der Ergebnisse anhand von konkreten Problemsituationen im Biosphärenpark Wienerwald beitragen. 


\section{LITERATURVERZEICHNIS}

Aarhus Konvention (1998): Übereinkommen über den Zugang zu Informationen, die Öffentlichkeitsbeteiligung in Entscheidungsverfahren und den Zugang zu Gerichten in Umweltangelegenheiten.

AGES (2008): Homepage der Österreichischen Agentur für Gesundheit und Ernährungssicherheit $\mathrm{GmbH}$ : Bewertung und Zulassung von Pflanzenschutzmitteln: http://www.ages.at/ages/ages-oesterreichische-agentur-fuer-gesundheit-undernaehrungssicherheit; abgerufen am 5. April 2008.

Albin, C. (1993): The Role of Fairness in Negotiations. Negotiation Journal 9(3): 223-244.

Alpenkonvention: http://www.alpenkonvention.org.

Anand, P. (2001): Procedural Fairness in Economic and Social Choice: Evidence from a Survey of Voters. Journal of Economic Psychology 22(2): 247-270. Elsevier.

Arbter, K.; Handler, M.; Purker, E.; Tappeiner, G. \& Trattnigg, R. (2005): Das Handbuch Öffentlichkeitsbeteiligung: Die Zukunft gemeinsam gestalten. Österreichische Gesellschaft für Umwelt und Technik (Hrsg.). Wien.

ARGE MTB NÖ (2008): http://www.mbike.at/mbike/noe/; abgerufen am 12.12.2008.

ARGE Wienerwald (2002): Machbarkeitsstudie Wienerwald - Eignung des Wienerwaldes für einen Nationalpark oder Biosphärenpark. Im Auftrag des Amtes der NÖ Landesregierung, Abteilung Forstwirtschaft und der Magistratsabteilung 49, Forstamt und Landwirtschaftsbetrieb der Stadt Wien.

Arnold, W. (2005): Schwarzwild: Hintergründe einer Explosion. In: Österreichs Weidwerk, 8-11.

Auer, M. (1982): Wiesengesellschaften im Wienerwald. Diplomarbeit, Universität für Bodenkultur, Wien.

AVL/Becker, B. et al. (2004): Detailplanung Biosphärenpark Wienerwald - Bereich Offenland: Studie im Auftrag von Biosphärenpark Wienerwald Management, Bearbeitung: AVL, Wien.

Bastian, O. \& Schreiber, K. F. (Hrsg.) (1999): Analyse und ökologische Bewertung der Landschaft. Gustav Fischer Verlag, Jena, Stuttgart.

Berg, H.-M. \& Zuna-Kratky, T. (1992): Die Brutvögel des Wienerwaldes: Eine kommentierte Artenliste (Stand August 1991). In: Vogelkundliche Nachrichten aus Ostösterreich, 1-11.

Berg, H.-M. \& Ranner, A. (1997): Rote Listen ausgewählter Tiergruppen Niederösterreichs. Vögel (Aves). Amt der NÖ Landesregierung/Abt. Naturschutz, Wien, 184 pp.

Berner Konvention: http://www.coe.int/

BEV (2008): Österreichische Karte 1:50.000 (ÖK 50).

Biosphärenpark Wienerwald: Homepage: http://bpww.at/home/ (abgerufen am 5. März 2009).

Bischoff, A.; Selle, K. \& Sinning, H. (2007): Kommunikation in Planungsprozessen: Eine Übersicht zu Formen, Verfahren und Methoden. Dortmund.

BMLFUW (2004): Österreichischer Aktionsplan zu gebietsfremden Arten (Neobiota). BMLFUW, Wien.

BMLFUW (2008): http://buergerservice.lebensministerium.at/article/articleview/24593/1/6766; abgerufen am 16.12.2008.

BMU (1995): Nationaler Umweltplan. Wien.

BMUJF (1998): Österreichische Strategie zur Umsetzung des Übereinkommens über die biologische Vielfalt. Wien. 
Bogner, A. \& Menz, W. (2005a): Expertenwissen und Forschungspraxis. In: Bogner, A.; Littig, B. \& Menz, W.: Das Experteninterview. Wiesbaden.

Bogner, A. \& Menz, W. (2005b): Das theoriegenerierende Experteninterview. In: Bogner, A.; Littig, B. \& Menz, W.: Das Experteninterview. Wiesbaden.

Bonner Konvention: http://www.cms.int/

Böck, C. \& Pötsch, M. (o. J.): Möglichkeiten zur Rettung von Wildtieren vor dem Mähtod. ÖAG, Österreichische Arbeitsgemeinschaft für Grünland und Futterbau.

Brandenburg, C. (2001): Erfassung und Modellierung von Besuchsfrequenzen in Erholungs- und Schutzgebieten - Anwendungsbeispiel Nationalpark Donau-Auen, Teilgebiet Lobau. Dissertation, Institut für Freiraumgestaltung und Landschaftspflege, Universität für Bodenkultur, Wien.

Brundtland, G. H. \& UNCED (1988): Our Common Future. Oxford University Press, Oxford.

Budil, B. (2000): Waldbauliches Behandlungskonzept für den Schwarzkiefern-Erholungswald der Stadt Mödling. Diplomarbeit, Institut für Waldbau, Universität für Bodenkultur, Wien.

CBD (1992): Übereinkommen über die biologische Vielfalt. http://www.cbd.int/.

Cornwall, A. \& Jewkes, R. (1995): What is Participatory Research? Social Science \& Medicine. Elsevier.

Council of Europe (2007): European Charter on Hunting and Biodiversity. Standing Committee of the Convention on the Conservation of European Wildlife and Natural Habitats. Strasbourg, 29.09.2007.

Daniels, S. E. \& Walker, G. B. (1997): Collaborative Learning and Land Management Conflict. In: Solberg, B. \& Miina, S. (eds.): Conflict Management and Public Participation in Land Management. Proceedings of the International Conference in Joensuu, Finland, 17-19 June 1996. EFI Proceedings No. 14, 1997: 37-60.

Deutsches MAB Nationalkomitee/Bundesamt für Naturschutz (1996): Kriterien für Anerkennung und Überprüfung von Biosphärenreservaten der UNESCO in Deutschland. Bundesamt für Naturschutz, Bonn.

Dvorak, M.; Ranner, A. \& Berg, H. M. (1993): Atlas der Brutvögel Österreichs: Ergebnisse der Brutvogelkartierung 1981-1985 der Österreichischen Gesellschaft für Vogelkunde. Umweltbundesamt, Wien.

Essl, F.; Egger, G.; Ellmauer, T.; \& Aigner, S. (2002): Rote Liste gefährdeter Waldbiotope Österreichs. In: Monographie Umweltbundesamt, Wien.

Essl, F. \& Rabitsch, W. (2002): Neobiota in Österreich. Umweltbundesamt, Wien.

Essl, F. \& Rabitsch, W. (2005): Neobiota in Österreich. In: Bundesministerium für Land- und Forstwirtschaft, Umwelt und Wasserwirtschaft (Hrsg.): Aliens: Neobiota in Österreich. Grüne Reihe des Lebensministeriums, Bd. 15: 28-47. Böhlau, Wien.

Europäische Kommission (2003): COST 341 - Habitat Fragmentation due to Transportation Infrastructure: Traffic and Wildlife. A European Handbook for Identifying Conflicts and Designing Solutions.

Fauna-Flora-Habitat-Richtlinie (1992): Richtlinie 92/43/EWG des Rates vom 21. Mai 1992 zur Erhaltung der natürlichen Lebensräume sowie der wildlebenden Tiere und Pflanzen.

Fischer, F. (1971): Die Grünflächenpolitik Wiens bis zum Ende des Ersten Weltkriegs. In: Schriftenreihe des Instituts für Städtebau, Raumplanung und Raumordnung, 14-22.

Flesch, P. \& Fraissl, C. (1994): Naturwaldreservate im Wienerwald. Vorstudie. MA 49 - Forstamt und Landwirtschaftsbetrieb der Stadt Wien (Hrsg.). 
Forstner, M.; Reimoser, F.; Hackl, J. \& Heckl, F. (2001): Kriterien und Indikatoren einer nachhaltigen Jagd. Monographien des Umweltbundesamtes, M-158. Umweltbundesamt, Wien.

Forstner, M.; Reimoser, F.; Lexer, W., Heckl, F. \& Hackl, J. (2006): Nachhaltigkeit der Jagd: Prinzipien, Kriterien, Indikatoren. Erweiterte Fassung. Umweltbundesamt (Hrsg.). avBuch, Wien.

Forstner, M.; Reimoser, F.; Lexer, W., Heckl, F. \& Hackl, S. (2003): Criteria and Indicators of Sustainable Hunting. Authors: English Translation of Monograph No. 158 (2001). Available only in digital format. http://www.biologischevielfalt.at/nachhaltige-nutzung/nachhaltige$\mathrm{jagd} /$.

Frank, G. \& Berg, H.-M. (2001): Verbreitung und Schutz des Schwarzstorches (Ciconia nigra) im Wienerwald. Unpublizierter Bericht von BirdLife Österreich, Wien, 1-32.

Friedrichs, J. (1980): Methoden empirischer Sozialforschung. Opladen.

Frühauf, J. (2005): Rote Liste der Brutvögel (Aves) Österreichs. In: Bundesministerium für Landund Forstwirtschaft, Umwelt und Wasserwirtschaft (Hrsg.): Rote Liste gefährdeter Tiere Österreichs (Teil 1). Grüne Reihe des Lebensministeriums, Bd. 14/1: 63-165. Böhlau, Wien.

Gläser, J. \& Laudel, G. (2004): Experteninterviews und qualitative Inhaltsanalyse. VS Verlag für Sozialwissenschaften / UTB, Wiesbaden.

Glitzner, I.; Beyerlein, P.; Brugger, C.; Egermann, F.; Paill, W.; Schlögel, B. \& Tataruch, F. (1999): Literaturstudie zu anlage- und betriebsbedingten Auswirkungen von Straßen auf die Tierwelt. Endbericht. Graz.

Glutz von Blotzheim, U. \& Bauer, K. (1973): Handbuch der Vögel Mitteleuropas, Bd. 5: Galliformes und Gruiformes. Wiesbaden.

Grabherr, G.; Koch, G.; Kirchmeier, H. \& Reiter, K. (1998): Hemerobie österreichischer Waldökosysteme. In: Veröffentlichung des Österreichischen MAB-Programmes. Universitätsverlag Wagner, Innsbruck.

Gross, E. \& Berg, H.-M. (2004): Position des NÖ Naturschutzbundes zum Jagdlichen Leitbild des Biosphärenparks Wienerwald.

Harborth, H. J. (1993): Dauerhafte Entwicklung statt globaler Selbstzerstörung: Eine Einführung in das Konzept der nachhaltigen Entwicklung. Edition sigma, Berlin.

Hartje, V.; Klaphake, A. \& Schliep, R. (2003): The International Debate on the Ecosystem Approach: Critical Review - International Actors - Obstacles and Challenges. BfN-Skripten 80. BfN, Bonn.

Häusler, W. (1980): Land zwischen Donau und Schöpfl. Jugend und Volk, Wien.

Heckl, F.; Lexer, W.; Vacik, H.; Wolfslehner, B. \& Hackl, J. (2003): Grundlagen für die Umsetzung des Ökosystemaren Ansatzes des Übereinkommens über die biologische Vielfalt: Aspekte des Schutzes und der nachhaltigen Nutzung der biologischen Vielfalt am Beispiel des österreichischen Waldes. Berichte des Umweltbundesamtes, Bd. BE 153. Umweltbundesamt $\mathrm{GmbH}$, Wien.

Herbold, H. (1992): Reaktionen von Rehen (Capreolus capreolus L., 1758) auf Störungen durch Menschen. Diss. Albert-Ludwigs-Univ. Freiburg.

Hirsch Hadorn, G. (2005): Anforderungen an eine Methodologie transdisziplinärer Forschung. In: Technikfolgenabschätzung - Theorie und Praxis, Nr. 2, Juni 2005: 44-49.

Hochbichler, E.; Budil, B. \& Köck (2001): Stadtwald Mödling, Erholungswald-Waldbewirtschaftung. 
Holzgang et al. (2001): Korridore für Wildtiere in der Schweiz. Schriftenreihe Umwelt Nr. 326. Bundesamt für Umwelt, Wald und Landschaft (BUWAL), Schweizerische Gesellschaft für Wildtierbiologie (SGW) \& Schweizerische Vogelwarte Sempach, Bern.

Holzner, W.; Bogner, D.; Geburek, T.; Tiefenbach, M. \& Zech, S. (2006): MOBI-e - Entwicklung eines Konzeptes für ein Biodiversitäts-Monitoring in Österreich. Studie im Auftrag des Lebensministerium. Wien.

IUCN (1994): IUCN Red List Categories. Prepared by the IUCN Species Survival Commission. As approved by the $40^{\text {th }}$ meeting of the IUCN Council. IUCN, Gland, Switzerland: 22.

IUCN (1999): IUCN Red List Criteria Review Provisional Report: Draft of the Proposed Changes and Recommendations. Species 31/32, 43-57.

IUCN (2000): Grundsatzerklärung der IUCN zur nachhaltigen Nutzung wildlebender Ressourcen. Weltkongress der IUCN, 10. Oktober, Amman, Jordanien. http://www.iucn.org/.

Kalchreuter, H., Guthörl, V. (1997): Wildtiere und menschliche Störungen - Ploblematik und Management. Informationen aus der Wildforschung. Verlag Dieter Hofrfmann, Mainz.

Kommission der Europäischen Gemeinschaften (2001): Weißbuch „Europäisches Regieren“. Brüssel.

Krajasits, C.; Mollay, U.; Neunteufel, G.; Spanring, C. \& Wach, I. (2004): Regionalanalyse Wienerwald. Österreichisches Institut für Raumplanung (ÖIR), Wien.

Lebersorger, P. \& Zeiler, H. (2005): Neobiota aus der Sicht der Jägerschaft. In: Bundesministerium für Land- und Forstwirtschaft, Umwelt und Wasserwirtschaft (Hrsg.): Aliens: Neobiota in Österreich. Grüne Reihe des Lebensministeriums, Bd. 15: 205-212. Böhlau, Wien.

Leputsch, S. (1997): Die Wiesen des Lainzer Tiergartens unter besonderer Berücksichtigung der Jagd-Tradition und der Erholungsnutzung. Diplomarbeit, Universität für Bodenkultur, Wien.

Lexer, W. (2004): Steps towards the Implementation of the Ecosystem Approach by the Example of Forests of Austria. In: Korn, H.; Schliep, R. \& Stadler, J. (eds.): Report of the International Expert Workshop on "Ways to Promote the Ideas behind CBD's Ecosystem Approach in Central and Eastern Europe", May 6-8, 2004, Vilm, Germany. Skripten des Bundesamtes für Naturschutz (BfN) (in print): 30-43.

Lexer, W. \& Reimoser, F. (2007): Assessment and Governance of Sustainable Hunting. WISPER Guidelines, revised Austrian PCls, and cross-sectoral assessment framework. Presentation at the IUCN ESUSG Workshop 'Using Wild Resources across Europe: Values and Governance', Sept. 15 2007, FIWI, Vienna.

Lexer, W.; Reimoser, F.; Hackl, J.; Heckl, F. \& Forstner, M. (2006): Criteria and Indicators of Sustainable Hunting - The Austrian Assessment Approach. In: Wildlife Biology in Practice, Vol. 1, No. 2 (2005): 163-183.

Lexer, W.; Reimoser, F.; Hackl, J.; Heckl, F. \& Forstner, M. (2005): Criteria and Indicators of Sustainable Hunting - The Austrian Assessment Approach. In: Wildlife Biology in Practice, Vol. 1/05: Proceedings of the XXVIth International Congress of the International Union of Game Biologists (IUGB), Sept. 1-6, 2003, Braga, Portugal.

Linser, S. (2001): Critical Analysis of the Basics for the Assessment of Sustainable Development by Indicators. PhD Thesis, University of Freiburg.

Löhr, L. (1990): Faustzahlen für den Landwirt

Loos, E. (2006): Richtlinie zur Erstellung naturschutzfachlicher Gutachten im Hinblick auf die Bewertung von Ersatz- und Ausgleichsmaßnahmen nach dem Salzburger Naturschutzgesetz. Amt der Salzburger Landesregierung, Naturschutzabteilung, Salzburg.

Lumpi, B. (1985): Die Bedeutung des Waldes in Kriegs- und Notzeiten, unter besonderer Berücksichtigung des Wienerwaldes. Dissertation, Universität für Bodenkultur, Wien. 
Manfredo, M. J.; Vaske, J. J. \& Sikorovski, L. (1996): Human Dimensions of Wildlife Management. In: Ewert, A. W. (ed.): Natural Resource Management: The Human Dimension. Westview Press, 53-72.

Mayer, H. (1974): Wälder des Ostalpenraumes. Gustav Fischer Verlag, Stuttgart.

Mayer, H. (2006): Interview und schriftliche Befragung. München.

Mayring, P. (1993): Qualitative Inhaltsanalyse. Grundlagen und Techniken. Deutscher Studienverlag, Weinheim.

MCPFE (1990): Entscheidungen und Beschlüsse der Ersten Ministerkonferenz zum Schutz der Wälder in Europa. Dezember 1990, Strassburg.

MCPFE (1993): Entscheidungen und Beschlüsse der Zweiten Ministerkonferenz zum Schutz der Wälder in Europa. 16.-17. Juni 1992, Helsinki..

MCPFE (1998): Entscheidungen und Beschlüsse der Dritten Ministerkonferenz zum Schutz der Wälder in Europa. Juni 1998, Lissabon..

MCPFE (2003): Entscheidungen und Beschlüsse der Vierten Ministerkonferenz zum Schutz der Wälder in Europa. 28.-30. April 2003, Wien.

Niederösterreichisches Jagdgesetz: LGBI. 6500-0 idgF.

NÖ NSG 2000: Niederösterreichisches Naturschutzgesetz 2000 idgF.

Niederösterreichisches Polizeistrafgesetz: LGBI. 4000 idgF.

Niesslein, E. (1975): Erholungsraum Wienerwald: Eine forstliche Raumordnungsstudie. Schriftenreihe der Österreichischen Gesellschaft für Raumforschung und Raumplanung. Springer Verlag.

ÖBf (2008): www.oebf.at.

ÖGUT (2003): Arbeitsblätter zur Partizipation Nr. 1: Checklisten für Rahmenbedingungen und Qualitätskriterien partizipativer Verfahren im öffentlichen Bereich.

ÖGUT (2004a): Arbeitsblätter zur Partizipation Nr. 2: Der Nutzen von Öffentlichkeitsbeteiligung aus der Sicht der Akteurlnnengruppen.

ÖGUT (2004b): Arbeitsblätter zur Partizipation Nr. 3: Grenzen, Stolpersteine und Instrumentalisierung von Öffentlichkeitsbeteiligung.

Österreichische Bundesregierung (2002): Die österreichische Strategie zur nachhaltigen Entwicklung. BMLFUW, 2002.

Ohl, C.; Stickler, T.; Lexer, W.; Beckenkamp, M.; Risnoveanu, G.; Geamana, N.; Fischer, A.; Fiorini, S.; Dumortier, M. \& Casaer, J. (2008): Governing Biodiversity: Procedural and Distributional Fairness in Complex Social Dilemmas (submitted).

Oitzinger, G.; Gruber, G.; Hiesberger, K.; Kuran, G.; Mayer, A.; Reimoser, F.; Neiss, H.; Pfalz, W. \& Wimmer, J. (2007): Ergebnisprotokoll „Jagdliche Einrichtungen in Kernzonen“. Österreichische Bundesforste AG, Purkersdorf.

Österreichische Bundesregierung (2002): Österreichische Strategie zur nachhaltigen Entwicklung. Wien. www.nachhaltigkeit.at/strategie/pdf/strategie020709_de.pdf.

Österreichisches Forstgesetz (2002): Österreichisches Forstgesetz 1975 in der Fassung 2002. BGBI. Nr. I 59/2002.

Pohl, Ch., 2004: Guidelines für die transdisziplinäre Forschung; http://www.transdisciplinarity.ch (09.05.2005)

Primack, R. B. (1998): Essentials of Conservation Biology. 2nd edition. Sinauer, Sunderland. 
Rauer, J. \& Gutleb, B. (1997): Der Braunbär in Österreich. In: Mongraphien des Umweltbundesamtes Bd. 110. Umweltbundesamt Wien, 1-54 \& Annex.

Reimoser, F. (1986): Wechselwirkungen zwischen Waldstruktur, Rehwildverteilung und Rehwildbejagbarkeit in Abhängigkeit von der waldbaulichen Betriebsform. Dissertation, Universität für Bodenkultur, 28. VWGÖ-Verlag, Wien.

Reimoser, F. (1996): Wildökologische Raumplanung für Schalenwildarten im Alpenraum. In: Sauteria, Salzburg, Bd. 8, 207-220.

Reimoser, F. (2001): Forest ungulate damage: Integrating wildlife and forest management practices. In: R. Field, R.J. Warren, H. Okarma, P.R. Sievert (eds.) Wildlife, Land, and People: Priorities for the 21st century. The Wildlife Society, Bethesd, Maryland, USA, 172-176.

Reimoser, F. (2001): Habitat - Waldschadendisposition - Wildschaden. In: Führer, E. \& Nopp, U. (Hrsg.): Ursachen, Vorbeugung und Sanierung von Waldschäden. Facultas Universitätsverlag, Wien, 136-184.

Reimoser, F.; Leitner, H.; Erber, J. \& Scheiderbauer, B. (2001): Wildökologische Raumplanung „Nationalpark Donau-Auen“ - Endbericht. Eigenverlag Nationalpark Donau-Auen, Orth an der Donau.

Reimoser, F. (2002): Wildökologische Raumplanung in Nationalparks und Natura 2000 Gebieten. In: Jagd und Natura 2000. Bundesanstalt für alpenländische Landwirtschaft (BAL) Gumpenstein des BMLFUW (Hrsg.), Irdning, Austria (ISBN 3-901980-70-9), S. 11-16.

Reimoser, F.; Lexer, W.; Forstner, M.; Hackl, J. \& Heckl, F. (2003): Kriterien und Indikatoren einer nachhaltigen Jagd. In: Zeitschrift für Jagdwissenschaft 49: 275-287.

Reimoser F, 2005: Freizeitaktivitäten und Wildtiere: Folgen für den Wald. In: Freizeitaktivitäten im Lebensraum der Alpentiere (P. Ingold, ed.). Haupt Verlag, Bern, S. 311-321.

Reimoser, F.; Reimoser, S. \& Klansek, E. (2006): Wild-Lebensräume - Habitatqualität, Wildschadenanfälligkeit, Bejagbarkeit. Zentralstelle Österreichischer Landesjagdverbände, Wien (ISBN 3-9501873-1-6).

Reimoser, F. \& Hackländer, K. (2007): Forstwege und Wildtiere. In: Der Anblick 4: 22-26.

Reimoser, F. \& Lexer, W. (2007): Assessment of Sustainable Use: Sustainable Hunting in Europe. Speech at the Intergroup Meeting "Sustainable Hunting, Biodiversity and Countryside Activities". European Parliament, Strasbourg, July 112007.

Rennings, K. (1994): Indikatoren für eine dauerhaft-umweltgerechte Entwicklung. MetzlerPoeschel, Stuttgart.

Salinger, M. (1972): Grundlagen für die waldbauliche Behandlung buchenreicher Waldgesellschaften auf Flysch-Standorten. Dissertation, Universität für Bodenkultur. Verlag Notring, Wien.

Scherzinger, W. (1996): Naturschutz im Wald: Qualitätsziele einer dynamischen Waldentwicklung. Eugen Ulmer, Stuttgart.

Schuster, A. (2005): Ausgewählte Neozoen: Vögel. In: Bundesministerium für Land- und Forstwirtschaft, Umwelt und Wasserwirtschaft (Hrsg.): Aliens: Neobiota in Österreich. Grüne Reihe des Lebensministeriums, Bd. 15: 121-132. Böhlau, Wien.

Sieber, J. \& Ulbel, G. (2002): Säugetiere. In: Perspektiven. N. J. Schmid Verlagsges.m.b.H., Wien, 75-82.

Spitzenberger, F. (2001): Die Säugetierfauna Europas. In: Bundesministerium für Land- und Forstwirtschaft, Umwelt und Wasserwirtschaft (Hrsg.): Grüne Reihe des Lebensministeriums, Bd. 13. 
Spitzenberger, F. (2005): Rote Liste der Säugetiere Österreichs (Mammalia). In: Zulka, K. P. (Red.): Rote Listen gefährdeter Tiere Österreichs. Grüne Reihe BMLFUW, Band 14/1, Böhlau Verlag, Wien, Köln, Weimar, 45-62.

SRU (1994): Umweltgutachten 1994: Für eine dauerhaft-umweltgerechte Entwicklung (Environmental Experts Report 1994).

Statistik Austria (2001): Agrarstrukturerhebung 1999. Betriebsstruktur. Schnellbericht 1.17. Statistik Austria, Wien.

Trumler, G. (1985): Das Buch vom Wienerwald: Landschaft, Kultur, Geschichte. Brandstätter Verlag, Wien.

Übereinkommen über die biologische Vielfalt (1992): Convention on Biological Diversity (CBD). http://www.cbd.int/; BGBI. Nr. 213/1995.

Umweltbundesamt (2005): Interaktive Internet-Plattform „Nachhaltige Jagd“. http://www.biologischevielfalt.at/nachhaltige-nutzung/nachhaltige-jagd/.

Umweltbundesamt (2008): Achter Umweltkontrollbericht. Umweltbundesamt GmbH, Wien.

Umweltbundesamt (Hrsg.) (2006): Pfefferkorn, W; Leitgeb-Zach, M; Heckl, F. \& Gottsberger, T.: Vielfalt statt Zwiespalt. Begleitfaden zum Mitgestalten von Lebensräumen - ein Beitrag zur Umsetzung der Biodiversitätskonvention. Logos, Berlin.

UNCED (1992a): Die Rio-Deklaration über Umwelt und Entwicklung. Generalversammlung der Vereinten Nationen, Document A/CONF.151/26 (Vol. I). http://www.un.org/documents/ga/ conf151/aconf15126-1annex1.htm.

UNCED (1992b): Agenda 21. Global Programme of Action for Sustainable Development. http://www.un.org/esa/sustdev/documents/agenda21/index.htm.

UNEP (2000): Decision 6 of the $5^{\text {th }}$ Conference of the Parties (COP 5) to the Convention on Biological Diversity: Ecosystem Approach. In: UNEP/CBD/COP/5/23.

UNEP (2004a): UNEP/CBD/COP/7/Decision VII/12. Decision VII/12 of the Conference of the Parties to the Convention on Biological Diversity, $7^{\text {th }}$ meeting, Kuala Lumpur, Malaysia, 9-20 Feb 2004: Sustainable Use. Annex II: Addis Ababa Principles and Guidelines of Sustainable Use of Biodiversity. In: UNEP CBD/COP/7/21.

UNEP (2004b): $7^{\text {th }}$ Conference of the Parties (COP 7) to the Convention on Biological Diversity: Ecosystem Approach - Annotations to the Rationales and Implementation Guidelines. In: UNEP/CBD/COP/7/21.

UNESCO \& MAB-ICC (1996): The Seville Strategy and the Statutory Framework of the World Network of Biosphere Reserves. UNESCO.

UNESCO \& MAB-ICC (2000): Seville+5 recommendations.

UNESCO \& MAB-ICC (2002): Guiding Principles for Projects on Biosphere Reserves.

Vogelschutz-Richtlinie (1979): Richtlinie 79/409/EWG des Rates vom 2. April 1979 über die Erhaltung der wildlebenden hogelbestände. lex.europa.eu/LexUriServ/LexUriServ.do?uri=CELEX:31979L0409:DE:NOT.

Völk, F.; Glitzner, I. \& Wöss, M. (2001): Kostenreduktion bei Grünbrücken durch deren rationellen Einsatz: Kriterien - Indikatoren - Mindeststandards. Straßenforschung, Heft 513. Bundesministerium für Verkehr, Innovation und Technologie, Wien. http://www.fsv.at.

Weinfurter, P. (1991): Die Bundesforste im Wienerwald. In: Österreichische Forstzeitung, 5-8.

Wiener Jagdgesetz (2001): Gesetz über die Regelung des Jagdwesens (Wiener Jagdgesetz). idF. LGBI. Nr. 34/2001.

Wiener Tierhaltegesetz (2005): LGBI. für Wien Nr. 39/1987; letzte Änderung Nr. 4/2005. 
Wildauer, L. (2006): Wildschwein (Sus scrofa) und Reh (Capreolus capreolus) in den Bezirken Zentral- und Ostösterreichs seit 1950: Abschuss- und Bestandesentwicklung, mögliche Einflussfaktoren, Wildschweinschäden in der Landwirtschaft. Diplomarbeit, Universität Wien.

Winna, F. (2002): Die 1000-jährige Geschichte des Wienerwaldes. Carl Gerold's Sohn Verlagsbuchhandlung KG, Wien

Zettel, H.; Schödl, S. \& Wiesbauer, H. (2004): Zur Kenntnis der Wildbienen (Hymenoptera: Apidae) in Wien, Niederösterreich und dem Burgenland (Österreich). In: Monographie der Österreichischen Gesellschaft für Entomofaunistik.

Zink, R. (2007): Machbarkeitsstudie „Habichtskauz-Wiederansiedlung im Biosphärenpark Wienerwald“. Studie im Auftrag der Österreichischen Zoo-Organisation (OZO), Forschungsinstitut für Wildtierkunde und Ökologie, Veterinärmedizinische Universität Wien.

Zulka, K. P. (Bearb.) (2005): Rote Listen gefährdeter Tiere Österreichs: Säugetiere, Vögel, Heuschrecken, Wasserkäfer, Netzflügler, Schnabelfliegen, Tagfalter. Checkliste, Gefährdungsanalysen, Handlungsbedarf. Bundesministerium für Land- und Forstwirtschaft, Umwelt und Wasserwirtschaft (Hrsg.). Böhlau, Wien.

Zulka, K. P.; Eder, E.; Höttinger, H. \& Weigand, E. (2001): Grundlagen zur Fortschreibung der Roten Listen gefährdeter Tiere Österreichs. Monographien des Umweltbundesamtes, M135. Umweltbundesamt, Wien. 